\title{
Dosage multiélémentaire des métaux et métalloïdes dans les milieux biologiques par ICP-MS : valeurs usuelles chez 100 témoins
}

\section{Biological fluids ICP-MS multi-elementary metal and metalloid determination : 100 healthy reference values}

Jean-Pierre GOULLÉ ${ }^{(1) *}$, Loïc MAHIEU $U^{(1)}$, Nicolas NEVEU ${ }^{(1)}$, Daniel BOUIGE ${ }^{(3)}$, Julien CASTERMANT ${ }^{(1)}$, Gilbert LAINE ${ }^{(1)}$, Marie-Pascale NOUVEAU ${ }^{(2)}$, Roseline GEHANNE ${ }^{(2)}$, Christian LACROIX ${ }^{(1)}$

(1) Laboratoire de Pharmacocinétique et de Toxicologie Cliniques, Groupe Hospitalier du Havre BP 24 - 76083 LE HAVRE CEDEX

(2) Service de Médecine Préventive, Groupe Hospitalier du Havre - BP 24 - 76083 LE HAVRE CEDEX

(3) Laboratoire de Biochimie, Groupe Hospitalier du Havre - BP 24 - 76083 LE HAVRE CEDEX

*Auteur à qui adresser la correspondance : Jean-Pierre GOULLÉ, Laboratoire de Pharmacocinétique et de Toxicologie Cliniques, Groupe Hospitalier du Havre - BP 24 - 76083 LE HAVRE CEDEX - FRANCE

Tél : 0232733223 - Fax : 0232733238 - E-mail : jgoulle@ch-havre.fr

Ce travail a été présenté au XI ${ }^{\mathrm{Ime}}$ Congrès annuel de la SFTA à Dinard

(Reçu le 3 décembre 2004 ; accepté le 31 décembre 2004)

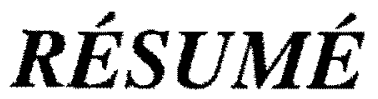

Une technique de dosage simultané d'une trentaine d'éléments minéraux dans le sang total, le plasma et les urines est décrite par plasma à couplage inductif relié à un détecteur de masse (ICP-MS). Elle permet de quantifier après une simple dilution en milieu acide, une trentaine de métaux et métalloüdes parmi : $\mathrm{Li}, \mathrm{Be}, \mathrm{B}, \mathrm{Al}, \mathrm{Mn}, \mathrm{Co}, \mathrm{Ni}, \mathrm{Cu}, \mathrm{Zn}, \mathrm{Ga}$, $\mathrm{Ge}, \mathrm{As}, \mathrm{Se}, \mathrm{Rb}, \mathrm{Sr}, \mathrm{Mo}, \mathrm{Pd}, \mathrm{Ag}, \mathrm{Cd}, \mathrm{Sn}, \mathrm{Sb}, \mathrm{Te}, \mathrm{Ba}, \mathrm{W}, \mathrm{Pt}, \mathrm{Hg}$, $T l, P b, B i, U$. Des valeurs de référence sont établies chez cent sujets témoins des deux sexes, indemnes de toute affection. En ce qui concerne les urines, les concentrations mesu-

\section{SUMMARY}

Multi-elementary whole blood, plasma and urine, quantification method using inductively coupled plasma mass spectrometry was developed (ICP-MS). A single acid dilution preparation procedure allows to quantify about thirty metals and metalloids : $\mathrm{Li}, \mathrm{Be}, \mathrm{B}, \mathrm{Al}, \mathrm{Mn}, \mathrm{Co}, \mathrm{Ni}, \mathrm{Cu}, \mathrm{Zn}, \mathrm{Ga}, \mathrm{Ge}$, $A s, S e, R b, S r, M o, P d, A g, C d, S n, S b, T e, B a, W, P t, H g, T l$, $\mathrm{Pb}, \mathrm{Bi}, U$. Normal values were determined among one hundred healthy volunteers biological fluids. As regards the urine samples, the results were corrected after enzymatic creatinine determination. Statistic treatment displays a non 
rées sont rapportées à la créatinine. L'analyse statistique montre que la distribution des résultats n'est pas gaussienne. Pour chaque paramètre et chaque milieu biologique, la valeur médiane et l'intervalle de mesure $5^{\text {tme }}$ percentile, $95^{\text {ine }}$ percentile sont calculés. L'examen des résultats montre que pour quelques éléments, un lien étroit unit les concentrations dans les différents milieux. L'analyse en composantes principales et l'examen des dendrogrammes montrent que certains métaux ont des comportements voisins. Pour bon nombre d'éléments, les valeurs usuelles sont inférieures à celles habituellement admises. Si cela s'explique pour certains d'entre eux, comme le plomb, par une baisse notable dans l'environnement, pour d'autres cela est du d̀ une. meilleure spécificité de la technique et à une amélioration de la détection limite. L'ICP-MS s'avère non seulement très puissante dans le cadre des recherches des causes de la mort, mais peut également trouver des applications en toxicologie clinique, pathologie professionnelle ou en surveillance environnementale.

\section{MOTS-CLÉS}

Plasma à couplage inductif, détecteur de masse, métaux, métalloïdes, liquides biologiques.

\section{Introduction}

Dans un travail précédent qui fait l'objet d'un correctif dans ce numéro (1), les auteurs avaient présenté la validàtion d'une technique de dosage multiélémentaire des métaux chez l'homme pour 24 éléments dans le sang total, pour 27 éléments dans le plasma et pour 29 éléments dans les urines par plasma à couplage inductif couplé à un détecteur de masse (ICP-MS). Si pour ceux qui sont quantifiés couramment, les valeurs normales font l'objet d'un certain consensus biologique, ce n'est pas le cas pour de nombreux éléments dont le dosage n'est accessible que par la torche à plasma, appareil d'usage récent en biologie clinique. Il convient également de rappeler que la plupart des valeurs usuelles ont été établies par spectrophotométrie d'absorption atomique avec flamme ou en mode électrothermique. Depuis deux ou trois décennies, ces valeurs fréquentes ont été revues à la baisse pour un grand nombre de métaux. Cette baisse s'explique par l'amélioration de la qualité des réactifs et des matériels de prélèvement, mais aussi et surtout grâce aux progrès réalisés dans la spécificité des mesures, en spectrophotométrie d'absorption atomique (SAA) électrothermique avec la correction du bruit de fond de type deutérium (correction du signal non spécifique) puis de type Zeeman (correction électromagnétique). L'apparition de l'ICP couplée à un détecteur optique (ICP-AES), et plus récemment dans sa version avec détecteur de masse (IPC-MS), sont susceptibles de diminuer également de manière sensible les normes établies pour de nombreux éléments. En effet, les performances de cette nouvelle gaussian distribution. For each parameter and each biological fluid, the median, and the range from the $5^{\text {th }}$ to the $95^{\text {th }}$ percentile were established. For some elements, a strong correlation links, the metal concentration in the different biological fluids. Many metals present lower reference values compared to the literature. For some of them, as lead, this is due to an important environmental decrease. For many others, the ICP-MS high specificity and low detection limit allows more precise determinations. This extremely effective equipment permits optimal clinical, forensic, occupational, and environmental toxicological evaluation of metal and metalloid status of each individual patient.

\section{KEY-WORDS}

Inductively coupled plasma, mass detector, metals, metalloids, biological fluids.

technique sont très supérieures à la SAA tant au plan de sa sensibilité que de sa spécificité, ainsi que nous l'avons montré (1). Aussi, il nous a semblé indispensable d'établir des valeurs normales pour les métaux et métalloïdes chez une population indemne de toute affection. Il est important de rappeler l'intérêt majeur de la torche à plasma qui permet, à partir d'une prise d'essai réduite, un dosage multiélémentaire simultané d'une trentaine d'éléments dans les milieux biologiques (plasma, sang total, urines, cheveux, tissus).

\section{Matériel et méthodes}

\section{Instrumentation :}

Il s'agit d'une torche à plasma de type ThermoOptek X Series couplée à un spectromètre de masse, modèle X7/CCT (ThermoOptek, Courtabœuf, France).

L'appareil est équipé d'une torche en quartz de $1,5 \mathrm{~mm}$, d'un nébuliseur concentrique en verre borosilicaté de $1 \mathrm{~mL}$ (type concentrique de Marque Glass Expansion Référence ThermoElemental 1201318) avec un débit d'échantillon de $0,85 \mathrm{~mL} / \mathrm{min}$, d'une chambre de nébulisation en quartz munie d'un refroidisseur à effet Peltier régulant sa température à $3^{\circ} \mathrm{C}$, et d'un passeur d'échantillons de type CETAC ASX-510. L'ensemble des données est enregistré sur une station informatique dotée du logiciel d'analyse PlasmaLab version 2.0 sous Windows NT. Les paramètres instrumentaux sont les suivants : puissance de la torche $1200 \mathrm{~W}$; débits d'argon : plasmagène $15 \mathrm{~L} / \mathrm{min}$, nébuliseur $0,95 \mathrm{~L} / \mathrm{min}$, auxiliaire $0,66 \mathrm{~L} / \mathrm{min}$; interface : cônes échantillon- 
neurs et écorceurs en nickel de diamètre respectifs de $1 \mathrm{~mm}$ et de $0,4 \mathrm{~mm}$; vide au niveau de l'interface : 1,9 mbar et du quadripôle : $1,6.10^{-7} \mathrm{mbar}$. Le dosage de la créatinine urinaire est réalisé par technique enzymatique sur Intégra 400 (Roche, Meylan, France).

\section{Sujets témoins :}

Du sang et des urines sont recueillis chez 100 volontaires adultes des deux sexes indemnes de toute affection à l'occasion de bilans réalisés sur des agents de l'établissement par le service de médecine préventive du personnel du Groupe Hospitalier du Havre (GHH Docteur M.P. Nouveau). Chaque témoin reçoit une information complète sur les modalités de prélèvement et les objectifs de ces analyses. Après signature de l'accord de participation, lors du bilan biologique, un tube supplémentaire de $7 \mathrm{~mL}$ de sang ainsi qu'un échantillon d'urines sont recueillis. Le sang est prélevé dans un tube sous vide de $7 \mathrm{~mL}$ hépariné pour éléments traces de type «Vacutainer $(B)$ référence 367735 (Becton Dickinson, Le Pont de Claix, France). Ce tube est également utilisé pour le zinc. La moitié de chaque prélèvement sanguin est décantée dans un tube en polystyrène de $5 \mathrm{~mL}$ puis centrifugée dans un délai de 2 heures après le prélèvement et le plasma immédiatement décanté. Le sang total, le plasma décanté sont congelés avant analyse. Les urines sont recueillies sur un flacon de $30 \mathrm{~mL}$ en polystyrène transparent muni d'un bouchon à vis en polypropylène et également congelées avant analyse. Pour des raisons pratiques, les mesures sont réalisées sur une miction et les résultats rapportés à la créatininurie. Les prélèvements sont transmis sans délai au laboratoire sous forme anonyme avec un questionnaire comportant les initiales du nom et du prénom, l'âge, la prise éventuelle de médicament (traitement contraceptif par exemple). Les sujets ayant un traitement médical contenant des métaux ou des oligo-éléments sont exclus. Le Tableau I montre la répartition des sujets par âge et par sexe. Les urines, la moitié du sang total ainsi que le plasma provenant de l'autre moitié du sang total après centrifugation sont congelés à $-27^{\circ} \mathrm{C}$ avant analyse.

\section{Réactifs :}

La source de plasma utilisée est de l'argon présentant un haut degré de pureté, supérieur à 99,999\% (Linde

Tableau I : Répartition des témoins par âge et par sexe.

\begin{tabular}{|c|c|c|c|c|c|c|}
\hline $\begin{array}{c}\text { Classe d'âge } \\
\text { (ans) }\end{array}$ & $<25$ & $\mathbf{2 5 - 3 4}$ & $\mathbf{3 5 - 4 4}$ & $\mathbf{4 5 - 5 4}$ & $\geq \mathbf{5 5}$ & Total \\
\hline Hommes & 2 & 16 & 11 & 11 & 6 & 46 \\
\hline Femmes & 9 & 17 & 17 & 11 & - & 54 \\
\hline
\end{tabular}

gas, Gargenville, France). L'eau utilisée est purifiée extemporanément sur Milli-Qpuus 185 (Millipore, St Quentin en Yvelines, France). L'acide nitrique à $65 \%$ de qualité suprapur, le 1-butanol, le triton X100 et les solutions multiélémentaires (multiélément standard solution VI contenant 30 éléments à 10 ou $100 \mathrm{mg} / \mathrm{L}$ ) proviennent de chez Merck (Darmstadt, Allemagne). Des étalons individuels de W, $\mathrm{Pt}, \mathrm{Pd}, \mathrm{Hg}, \mathrm{Sb}, \mathrm{Sn}, \mathrm{Ge}$ (CPI international, Amsterdam, Hollande) sont ajoutés à ces solutions multiélémentaires. L'étalonnage interne de l'appareil est réalisé avec une solution de Rhodium (CPI international). La validité des dosages est vérifiée pour le plasma, le sang total et les urines par des contrôles titrés par ICP-MS Seronorm niveau 1 (Trace elements serum level 1 - réf. 201405 ; Trace elements whole blood 2 - réf. 201607 ; Trace elements urine réf. 201207 ; SERO AS, Billingstade, Norvège). Le laboratoire participe au contrôle qualité ICP-MS canadien (Programme de comparaisons interlaboratoires, Sainte Foy, Canada) qui adresse trois fois par an à ses adhérents du sang total, des urines et des cheveux.

\section{Préparation des gammes d'étalonnage :}

Les gammes d'étalonnage permettant le dosage multiélémentaire dans le plasma sont réalisées en solution aqueuse, directement dans la solution diluante. En fait, l'étalonnage se fait par l'intermédiaire d'une gamme de concentrations en 8 points, préparée dans une solution d'acide nitrique $0,144 \mathrm{M}$, s'échelonnant de 0 à $24 \mu \mathrm{g} / \mathrm{L}$ $(0-0,5-1-2-3-6-12$ et $24 \mu \mathrm{g} / \mathrm{L})$ ou de 0 à $240 \mu \mathrm{g} / \mathrm{L}(0-5-$ 10-20-30-60-120 et $240 \mu \mathrm{g} / \mathrm{L}$ ) selon l'élément. En ce qui concerne le sang total, le dosage multiélémentaire est effectué par la méthode des ajouts dosés, autrement dit par une gamme de concentrations dans la matrice considérée, préparée à l'aide d'un pool de sang. Une gamme d'ajouts dosés est réalisée, s'échelonnant de $X$ à $\mathrm{X}+24 \mu \mathrm{g} / \mathrm{L}(\mathrm{X}, \mathrm{X}+0,5, \mathrm{X}+1, \mathrm{X}+3, \mathrm{X}+6, \mathrm{X}+12$ et $X+24 \mu g / L)$ ou de $X$ à $X+240 \mu g / L(X, X+5, X+10$, $X+30, X+60, X+120$ et $X+240 \mu g / L)$ selon les éléments. Pour les urines deux dilutions différentes sont utilisées en fonction de l'élément :

- au dixième pour le $\mathrm{B}, \mathrm{Se}, \mathrm{Rb}, \mathrm{Sr}, \mathrm{Pb}$, dans ce cas on utilise une gamme identique à celle du plasma, - au cinquième pour le $\mathrm{Li}, \mathrm{Be}, \mathrm{Al}, \mathrm{V}, \mathrm{Mn}, \mathrm{Co}, \mathrm{Ni}, \mathrm{Cu}$, $\mathrm{Zn}, \mathrm{Ga}, \mathrm{Ge}, \mathrm{As}, \mathrm{Mo}, \mathrm{Pd}, \mathrm{Cd}, \mathrm{Sn}, \mathrm{Sb}, \mathrm{Te}, \mathrm{Ba}, \mathrm{W}, \mathrm{Pt}, \mathrm{Hg}$, $\mathrm{Tl}, \mathrm{Bi}, \mathrm{U}$. En raison des effets de matrice, il est fait appel à une gamme selon la méthode des ajouts dosés, à partir d'un pool d'urines, dans les mêmes conditions et aux mêmes concentrations que pour le sang total.

\section{Préparation des échantillons :}

Les échantillons plasmatiques sont préparés par dilu- 
tion au dixième, dans une solution aqueuse composée d'acide nitrique $0,144 \mathrm{M}$, de $0,5 \%$ de 1-butanol et de triton $0,01 \%(\mathrm{v} / \mathrm{v})$, dans des tubes en polypropylène. Les échantillons de sang, préalablement congelés à $27^{\circ} \mathrm{C}$ puis décongelés, sont dilués au dixième dans une solution aqueuse composée d'acide nitrique $0,144 \mathrm{M}$, de $0,5 \%$ de 1-butanol et de triton $0,1 \%$ (v/v) dans des tubes en polypropylène. Une fois l'hémolyse achevée, les mélanges sont centrifugés à 4000 tours $/ \mathrm{min}$. Les urines sont préparées par dilution au 1:10 [acide nitrique $0,216 \mathrm{M}, 0,5 \%$ de 1 -butanol, triton $0,01 \%$ $(\mathrm{v} / \mathrm{v})]$ pour les éléments suivants : $\mathrm{B}, \mathrm{Se}, \mathrm{Rb}, \mathrm{Sr}, \mathrm{Pb}$ (gamme aqueuse). Les urines sont diluées au $1: 5$ [acide nitrique $0,216 \mathrm{M}, 0,5 \%$ de 1-butanol, triton $0,01 \%(\mathrm{v} / \mathrm{v})]$ pour les autres éléments : $\mathrm{Li}, \mathrm{Be}, \mathrm{Al}, \mathrm{V}$, $\mathrm{Mn}, \mathrm{Co}, \mathrm{Ni}, \mathrm{Cu}, \mathrm{Zn}, \mathrm{Ga}, \mathrm{Ge}, \mathrm{As}, \mathrm{Mo}, \mathrm{Pd}, \mathrm{Cd}, \mathrm{Sn}, \mathrm{Sb}$, $\mathrm{Te}, \mathrm{Ba}, \mathrm{W}, \mathrm{Pt}, \mathrm{Hg}, \mathrm{Tl}, \mathrm{Bi}, \mathrm{U}$ (ajouts dosés). La quantité d'échantillon sanguin, plasmatique ou urinaire nécessaire est de $0,4 \mathrm{ml}$.

\section{Les outils mathématiques et statistiques : Établissement des valeurs normales :}

Pour établir des valeurs dites de référence, il est nécessaire d'indiquer une valeur moyenne ainsi qu'un indicateur de dispersion. On utilise communément la moyenne arithmétique et l'écart type. Cependant l'apparition de valeurs s'écartant nettement des autres pèse considérablement sur les calculs. Pour cette raison nous avons utilisé les percentiles, indicateurs beaucoup plus robustes aux valeurs extrêmes. La médiane est donnée à titre indicatif $(2,3)$.

\section{Outils d'aide à l'interprétation des résultats :}

Un des objectifs est de comparer les concentrations d'un ou de plusieurs éléments dans divers milieux. L'analyse des résultats doit donc tenir compte du caractère multiparamétrique des mesures obtenues. Elle nécessite de faire appel à différents tests statistiques :

- L'analyse de corrélation qui permet d'évaluer l'existence d'un lien entre la concentration d'un élément et celle d'un autre, ou d'un même élément donné dans deux milieux différents. Afin de se prémunir contre l'apparition de corrélations fictives dues à des valeurs s'écartant nettement des autres, il est fait appel à la méthode de Spearman $(2,3)$ qui utilise le rang relatif des résultats et non plus les valeurs elles-mêmes.

- L'analyse en composantes principales est une représentation graphique qui met en évidence le comportement des variables les unes par rapport aux autres $(2,3)$. Cette analyse est réalisée milieu par milieu en excluant les éléments pour lesquels plus de $30 \%$ des témoins ont une concentration inférieure à la limite de détection. La représentation graphique de l'analyse en com- posantes principales est la projection dans un espace bidimensionnel de données elles-mêmes plongées dans un espace ayant 21 dimensions, c'est à dire autant de dimensions qu'il y a de variables. La matrice des corrélations entre ces données sert à définir la métrique nécessaire au calcul des distances entre les variables. Ce traitement mathématique est effectué en travaillant sur le rang des mesures et non sur leur valeur absolue, ce qui permet d'éliminer le poids considérable que peuvent avoir des valeurs extrêmes.

- L'analyse par classification hiérarchique ascendante permet d'étudier également chaque milieu indépendamment $(2,3)$ comme pour l'analyse en composantes principales. Seuls les éléments présentant moins de $30 \%$ d'individus inférieurs à la limite de détection sont pris en compte. La classification hiérarchique ascendante utilise aussi le rang des valeurs individuelles et non leur concentration. En revanche, contrairement à l'analyse en composantes principales, la classification hiérarchique ascendante conserve la totalité des dimensions de l'espace soit autant que de paramètres pris en compte. Il s'agit donc d'une analyse plus fine que la précédente. Elle consiste à calculer la distance entre chaque point représentant un élément, puis l'on agrège deux à deux les points les plus proches pour en former un nouveau, issu du centre de gravité entre ces deux points. On recommence ensuite l'opération avec le point le plus proche de ce dernier et ainsi de suite. On obtient ainsi une arborescence ou dendogramme traduisant la proximité relative de chaque élément par rapport aux autres. La technique utilisée est fondée sur le calcul du carré de la distance euclidienne entre chaque point, selon la méthode de Ward (2).

\section{Cas des mesures inférieures à la limite de détec- tion :}

Dans les trois matrices, sang total, plasma, urines, les dosages montrent pour certains éléments, selon une proportion variable, un signal inférieur à la limite de détection établie. Nous avons alors, selon une règle communément admise pour ce type d'analyse attribué une valeur arbitraire égale à la moitié de la limite de détection (3).

\section{Résultats}

Ils sont exprimés en $\mu \mathrm{g} / \mathrm{L}(=\mathrm{ng} / \mathrm{mL}$ ou $\mathrm{ppb})$ pour le plasma (Tableau II) et le sang total (Tableau III) et rapportés à la créatinine pour les urines soit en $\mu \mathrm{g} / \mathrm{g}$ de créatinine (Tableau IV). Pour chaque milieu, sont indiqués par élément : la valeur médiane, la dispersion des mesures, ainsi que les valeurs pour $90 \%$ de la population étudiée du $5^{\star}$ au $95^{\circ}$ percentile, ou la limite supé- 
Tableau II : Valeurs usuelles dans le plasma chez 100 sujets témoins des deux sexes. Les résultats sont exprimés en $\mu \mathrm{g} / L$ ou $n g / L$

\begin{tabular}{|c|c|c|c|c|c|}
\hline & LD & LQ & $\begin{array}{c}\text { \% } \\
\text { Ld }\end{array}$ & médiane & $\begin{array}{c}\text { Dispersion } \\
\text {-95 percentile }\end{array}$ \\
\hline Lithium & 0,2 & 0,6 & 0 & 3,4 & $1,8-18,8$ \\
\hline Béryllium & 0,03 & 0,10 & 63 & 0,015 & $0,015-0,103$ \\
\hline Bore & 1,3 & 4,2 & 0 & 36 & $19-79$ \\
\hline Aluminium & 2,3 & 7,7 & 45 & 3,1 & $1,2-17,3$ \\
\hline Vanadium & & & & & \\
\hline Chrome & & & & & \\
\hline Manganèse & 0,02 & 0,08 & 0 & 1,12 & $0,63-2,26$ \\
\hline Cobalt & 0,09 & 0,28 & 0 & 0,49 & $0,30-1,02$ \\
\hline Nickel & 0,08 & 0,28 & 23 & 2,20 & $0,04-5,31$ \\
\hline Cuivre & 0,1 & 0,5 & 0 & 1075 & $764-2023$ \\
\hline Zinc & 0,6 & 2,1 & 0 & 726 & $551-925$ \\
\hline Gallium & 0,16 & 0,52 & 0 & 6,24 & $5,03-8,82$ \\
\hline Germanium & 0,03 & 0,09 & 0 & 5,06 & $3,70-6,17$ \\
\hline Arsenic & 0,04 & 0,13 & 0 & 6,2 & $4,4-14,2$ \\
\hline Sélénium & 0,7 & 2,0 & 0 & 112 & $79-141$ \\
\hline Rubidium & 0,01 & 0,05 & 0 & 147,8 & $101-358$ \\
\hline Strontium & 0,02 & 0,05 & 0 & 28,8 & $18-75$ \\
\hline Molybdène & 0,03 & 0,09 & 0 & 0,96 & $0,67-1,68$ \\
\hline Palladium & & & & & \\
\hline Argent & & & & $\vdots$ & \\
\hline Cadmium & 0,01 & 0,03 & 3 & 0,03 & $0,01-0,05$ \\
\hline Etain & 0,3 & 1,0 & 1 & 1,82 & $0,15-2,70$ \\
\hline Antimoine & 0,006 & 0,019 & 5 & 0,11 & $0,03-0,15$ \\
\hline Tellure & 0,01 & 0,04 & 1 & 0,057 & $0,02-0,13$ \\
\hline Baryum & 2,1 & 7,0 & 0 & 111 & $90-154$ \\
\hline Tungstène & 0,19 & 0,62 & 32 & 0,24 & $0,09-0,75$ \\
\hline Platine & 0,01 & 0,02 & 0 & 0,39 & $0,016-0,92$ \\
\hline Mercure & & & & & \\
\hline Thallium & 0,003 & 0,012 & 0 & 0,06 & $0,01-0,24$ \\
\hline Plomb & 0,03 & 0,10 & 30 & 0,062 & $0,014-0,25$ \\
\hline Bismuth & 0,004 & 0,012 & 64 & 0,002 & $0,002-0,401$ \\
\hline Uranium & 0,001 & 0,002 & 0 & 0,007 & $0,004-0,011$ \\
\hline
\end{tabular}

LD : Limite de détection - LQ : Limite de quantification

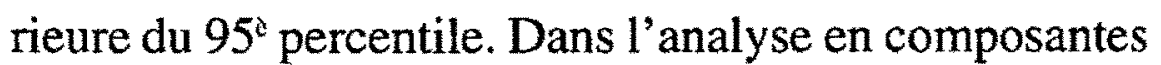
principales, 21 éléments ont été retenus pour chaque milieu (sang total, plasma, urines), c'est à dire ceux pour lesquels au moins $70 \%$ de la population présentait des résultats supérieurs à la limite de détection (Figures 1,2,3). Ce critère a également été retenu en ce qui concerne l'analyse par classification hiérarchique ascendante (Figures 1,2,3).
Tableau III : Valeurs usuelles dans le sang total chez 100 sujets témoins des deux sexes. Les résultats sont exprimés en $\mu \mathrm{g} / \mathrm{L}$ ou $\mathrm{ng} / \mathrm{mL}$.

\begin{tabular}{|c|c|c|c|c|c|}
\hline & LD & LQ & $\begin{array}{c}\text { \% } \\
\text { LD }\end{array}$ & médiane & $\begin{array}{c}\text { Dispersion } \\
\text {-95 percentile }\end{array}$ \\
\hline Lithium & & & & & \\
\hline Béryllium & 0,04 & 0,14 & 79 & 0,02 & $0,02-0,09$ \\
\hline Bore & 1,3 & 4,4 & 0 & 26 & $14-44$ \\
\hline Aluminium & 2,6 & 8,1 & 30 & 1,3 & $1,28-6,35$ \\
\hline Vanadium & & & & & \\
\hline Chrome & & & & & \\
\hline Manganèse & 0,03 & 0,09 & 0 & 7,6 & $5,0-12,8$ \\
\hline Cobalt & 0,02 & 0,06 & 3 & 0,25 & $0,04-0,64$ \\
\hline Nickel & 0,19 & 0,63 & 18 & 2,1 & $0,09-4,18$ \\
\hline Cuivre & & & & & \\
\hline Zinc & & & & & \\
\hline Gallium & 0,01 & 0,04 & 0 & 3,5 & $2,65-4,71$ \\
\hline Germanium & 0,05 & 0,17 & 0 & 16 & $10,8-19,5$ \\
\hline Arsenic & 0,03 & 0,11 & 0 & 5,0 & $2,6-17,8$ \\
\hline Sélénium & 0,5 & 1,6 & 0 & 119 & $89-154$ \\
\hline Rubidium & 0,02 & 0,06 & 0 & 1680 & $1289-2358$ \\
\hline Strontium & 0,01 & 0,02 & 0 & 16 & $9-41$ \\
\hline Molybdène & 0,02 & 0,07 & 0 & 2,9 & $0,77-7,86$ \\
\hline Palladium & 0,01 & 0,04 & 11 & 0,08 & $0,01-0,71$ \\
\hline Argent & 0,08 & 0,27 & 0 & 1,4 & $0,69-4,51$ \\
\hline Cadmium & 0,01 & 0,04 & 0 & 0,31 & $0,15-2,04$ \\
\hline Etain & 0,02 & 0,07 & 0 & 1,1 & $0,11-1,75$ \\
\hline Antimoine & 0,01 & 0,03 & 0 & 0,08 & $0,05-0,13$ \\
\hline Tellure & 0,02 & 0,06 & 0 & 0,16 & $0,11-0,45$ \\
\hline Baryum & 0,06 & 0,19 & 0 & 59 & $46,4-77,6$ \\
\hline Tungstène & 0,01 & 0,03 & 49 & 0,006 & $0,004-0,082$ \\
\hline Platine & 0,004 & 0,01 & 75 & 0,002 & $0,002-0,010$ \\
\hline Mercure & 0,08 & 0,26 & 0 & 3,0 & $0,94-8,13$ \\
\hline Thallium & 0,002 & 0,005 & 0 & 0,02 & $0,011-0,035$ \\
\hline Plomb & 0,02 & 0,07 & 0 & 26 & $11,4-62,8$ \\
\hline Bismuth & 0,002 & 0,007 & 68 & 0,001 & $0,001-0,007$ \\
\hline Uranium & 0,001 & 0,002 & 0 & 0,004 & $0,002-0,006$ \\
\hline
\end{tabular}

LD : Limite de détection - LQ : Limite de quantification

\section{Discussion}

En ce qui concerne les valeurs usuelles des médianes et des dispersions, la répartition des mesures montre qu'aucun élément ne présente une distribution gaussienne. Cette constatation est conforme à ce que l'on connaît de la répartition des concentrations d'un métal chez l'homme (4-6). Pour cette raison, l'expression des résultats sous forme de valeur médiane, avec les percentiles pour $90 \%$ de la population (percentile $5 \%$ à $95 \%$ ), nous semble beaucoup plus pertinente que la 
Tableau IV : Valeurs usuelles dans les urines chez 100 sujets témoins des deux sexes. Les résultats sont exprimés en $\mu \mathrm{g} / \mathrm{g}$ de créatinine.

\begin{tabular}{|c|c|c|c|c|c|}
\hline & LD & LQ & $\begin{array}{c}\text { \% } \\
\text { LD }\end{array}$ & médiane & $\begin{array}{c}\text { Dí.95 persion } \\
\text { percentile }\end{array}$ \\
\hline Lithium & 0,01 & 0,02 & 0 & 12 & $4,6-219$ \\
\hline Béryllium & 0,02 & 0,05 & 81 & 0,01 & $0,008-0,042$ \\
\hline Bore & 0,24 & 0,82 & 0 & 647 & $282-2072$ \\
\hline Aluminium & 0,32 & 1,1 & 14 & 1,9 & $0,16-11,2$ \\
\hline Vanadium & 0,04 & 0,15 & 0 & 3,3 & $1,4-10,2$ \\
\hline Chrome & & & & & \\
\hline Manganèse & 0,01 & 0,03 & 0 & 0,31 & $0,11-1,32$ \\
\hline Cobalt & 0,02 & 0,06 & 0 & 0,30 & $0,16-1,14$ \\
\hline Nickel & 0,06 & 0,21 & 4 & 1,8 & $0,59-4,06$ \\
\hline Cuivre & 0,05 & 0,16 & 0 & 6,9 & $4,3-12,1$ \\
\hline Zinc & 0,51 & 1,7 & 0 & 195 & $44-499$ \\
\hline Gallium & 0,01 & 0,02 & 1 & 0,07 & $0,02-0,28$ \\
\hline Germanium & 0,02 & 0,08 & 0 & 2,0 & $1,17-3,37$ \\
\hline Arsenic & 0,03 & 0,10 & 0 & 19 & $2,3-161$ \\
\hline Sélénium & 0,65 & 2,2 & 0 & 20 & $10,5-45,5$ \\
\hline Rubidium & 0,02 & 0,06 & $0 \cdot$ & 1211 & $433-2698$ \\
\hline Strontium & 0,004 & 0,01 & 0 & 90 & $20-413$ \\
\hline Molybdène & 0,06 & 0,20 & 0 & 20 & $7-50$ \\
\hline Palladium & 0,14 & 0,45 & 66 & 0,07 & $0,07-0,64$ \\
\hline Argent & & & & & \\
\hline Cadmium & 0,01 & 0,02 & 0 & 0,16 & $0,06-0,79$ \\
\hline Etain & 0,01 & 0,03 & 1 & 0,32 & $0,05-2,28$ \\
\hline Antimoine & 0,003 & 0,01 & 0 & 0,04 & $0,02-0,08$ \\
\hline Tellure & 0,02 & 0,07 & 0 & 0,23 & $0,10-0,52$ \\
\hline Baryum & 0,02 & 0,07 & 0 & 0,89 & $0,17-3,85$ \\
\hline Tungstène & 0,01 & 0,04 & 22 & 0,03 & $0,01-0,09$ \\
\hline Platine & 0,004 & 0,01 & 37 & 0,005 & $0,002-0,036$ \\
\hline Mercure & 0,29 & 0,95 & 15 & 0,59 & $0,14-2,21$ \\
\hline Thallium & 0,14 & 0,47 & 47 & 0,15 & $0,07-0,84$ \\
\hline Plomb & 0,02 & 0,06 & 10 & 0,55 & $0,01-2,14$ \\
\hline Bismuth & 0,001 & 0,003 & 44 & 0,001 & $0,0005-0,009$ \\
\hline Uranium & 0,0003 & 0,001 & 8 & 0,002 & $0,0002-0,008$ \\
\hline
\end{tabular}

LD : Limite de détection - LQ : Limite de quantification

moyenne arithmétique et les 2 écart-types. En effèt, en cas de grande dispersion des valeurs, la valeur absolue de l'écart type est importante. D'autre part, dans ce cas, la moyenne est nettement affectée par les valeurs extrêmes. Enfin, quand on retranche deux écart-types, la limite inférieure peut devenir négative. Le lithium urinaire constitue un bon exemple (Tableau V). Ce mode d'expression (médiane, percentiles pour $90 \%$ de la population), s'avère également pertinent si une partie importante de la population témoin présente des valeurs inférieures à la limite de détection comme pour l'aluminium plasmatique avec $45 \%$ des mesures de la population témoin inférieures à la limite de détection (Tableau V). Par ailleurs, une concentration normale supérieure fixée à $17,3 \mu \mathrm{g} / \mathrm{L}$ pour ce métal répond parfaitement aux préoccupations toxicologiques et est à l'évidence mieux adaptée que celle de $63 \mu \mathrm{g} / \mathrm{L}$, qui est une valeur nettement augmentée. L'analyse des résultats montre que pour de nombreux éléments, les concentrations mesurées chez les cent témoins sont inférieures aux valeurs normales communément admises (7). C'est le cas pour l'antimoine et le manganèse dans le sang total, le plasma et les urines; pour le cadmium et le thallium dans le sang total et les urines ; pour le bore dans le plasma et les urines; pour l'argent, le bismuth, l'étain, le plomb, le rubidium dans le sang total ; pour le baryum, le cobalt, le mercure, le tellure dans les urines.

Nous examinerons successivement l'analyse des corrélations entre les divers milieux biologiques, puis entre éléments, enfin les composantes principales et la classification hiérarchique ascendante. Pour les corrélations entre milieux biologiques, on admet habituellement que la recherche d'une relation entre la concentration d'un élément dans le sang, le plasma et les urines doit être considérée comme positive au seuil de signification de $1 \%$. Cependant, afin de se mettre à l'abri de corrélations accidentelles n'apparaissant que par pur hasard, nous avons retenu les corrélations dont le risque de première espèce est inférieur à $1 \%$. C'est le cas pour le cobalt, le bore, l'arsenic, le strontium. Cette constatation est utile car le dosage dans l'une des trois matrices sera suffisant pour renseigner sur le statut de l'un de ces quatre éléments dans l'organisme.

En ce qui concerne les corrélations entre éléments, nous remarquons que pour certains d'entre eux, très proches dans la table périodique, il existe un lien entre leur concentration dans un milieu biologique donné. Il est logique que leur comportement au sein de l'organisme soit proche. C'est le cas pour l'étain et l'antimoine aussi bien dans le plasma que dans le sang total.

Pour ce qui est de l'analyse en composantes principales et de la classification hiérarchique ascendante, il convient d'être prudent dans l'interprétation du graphique pour l'analyse en composantes principales, car des points peuvent avoir une projection proche mais être en réalité très distants dans l'espace. Cependant, l'intérêt de cette approche est de réunir sur un même graphique les comportements de tous les éléments et d'avoir une vision globale sur l'ensemble des paramètres pour chaque milieu biologique. Ainsi dans les urines, en dehors de toute pathologie (atteinte rénale, exposition à un métal), il y a incontestablement un effet lié à la filtration rénale, une élimination augmentée 
Analyse en Composantes Principales dans le sang total

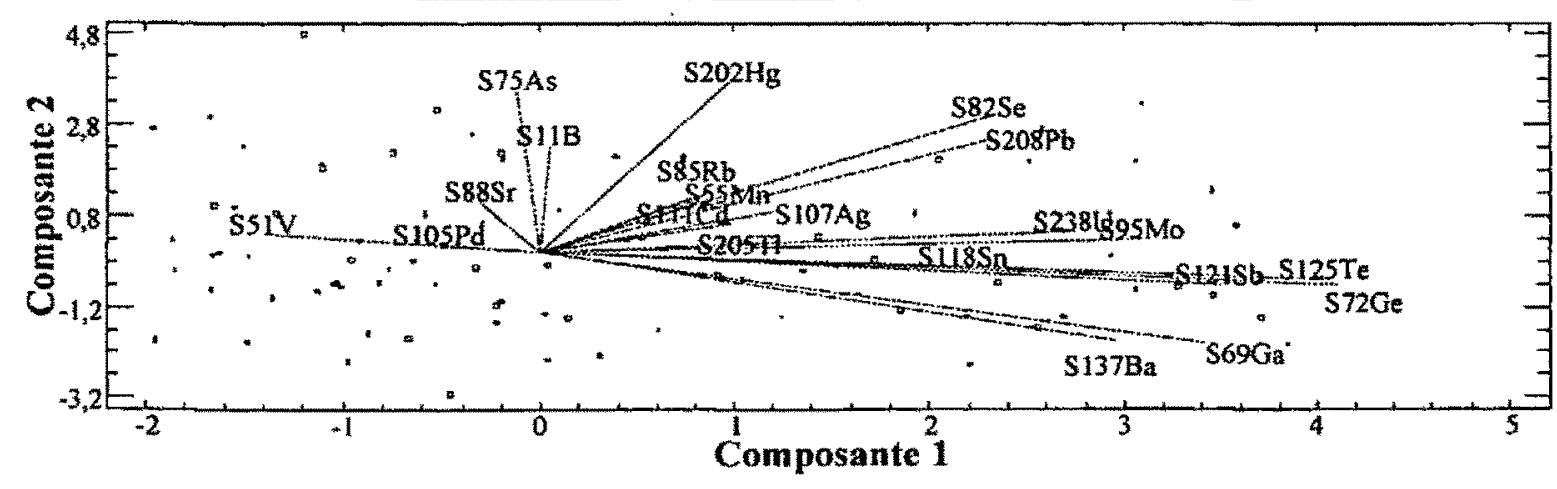

Classification hiérarchique ascendante dans le sang total

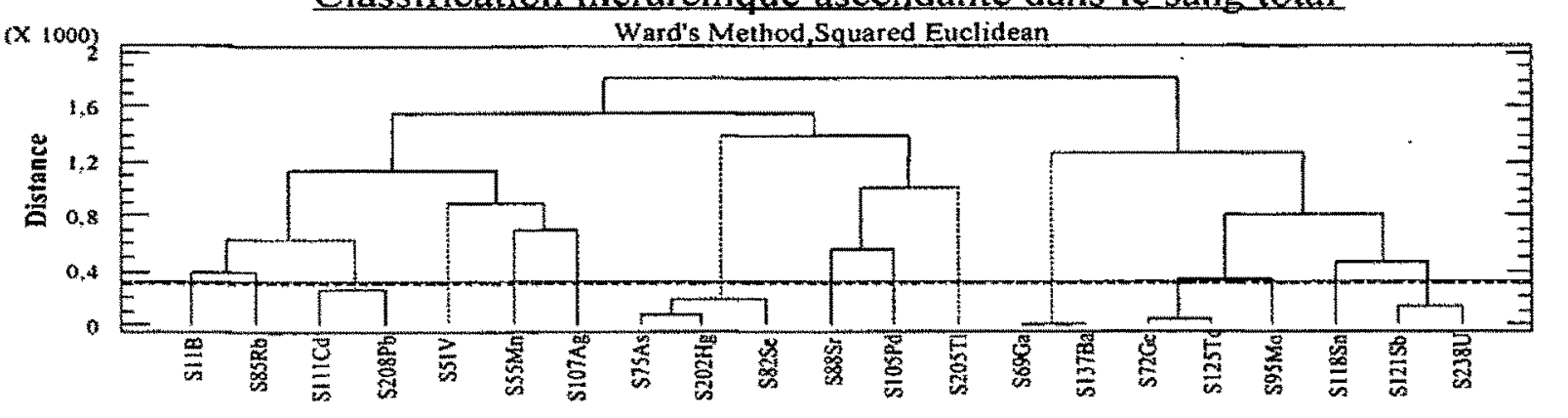

Figure I: Analyse en composantes principales et classification hiérarchique ascendante dans le sang total.
Figure 2 : Analyse en composantes principales et classification hiérarchique ascendante dans le plasma.
Analyse en Composantes Principales dans le plasma

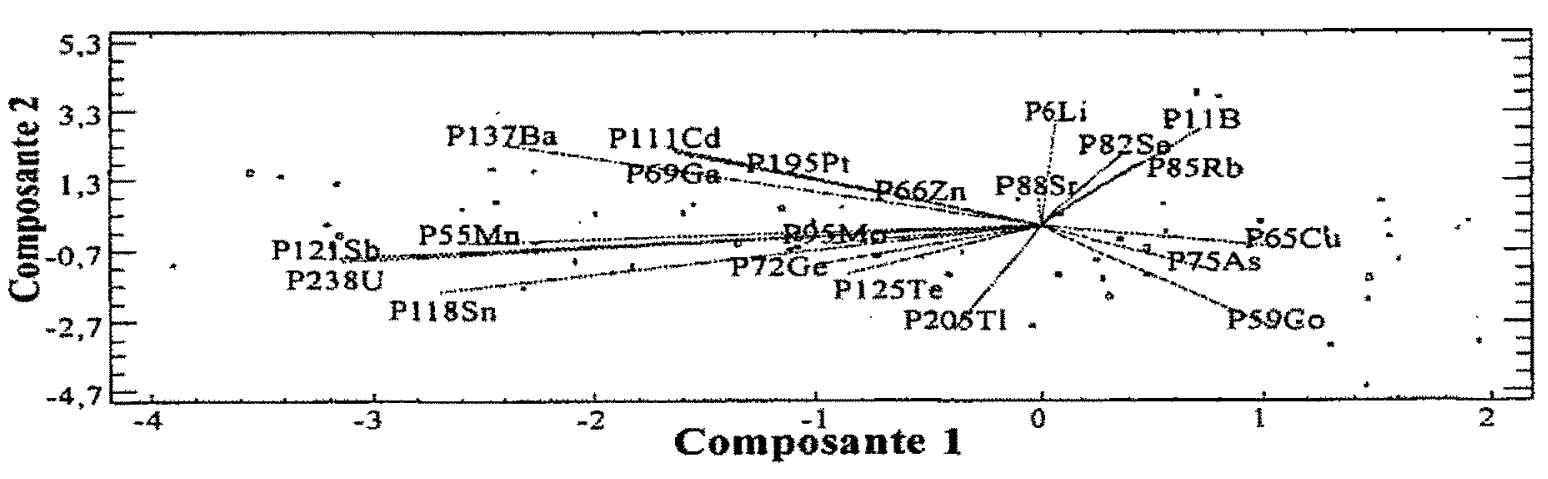

Classification hiérarchique ascendante dans le plasma

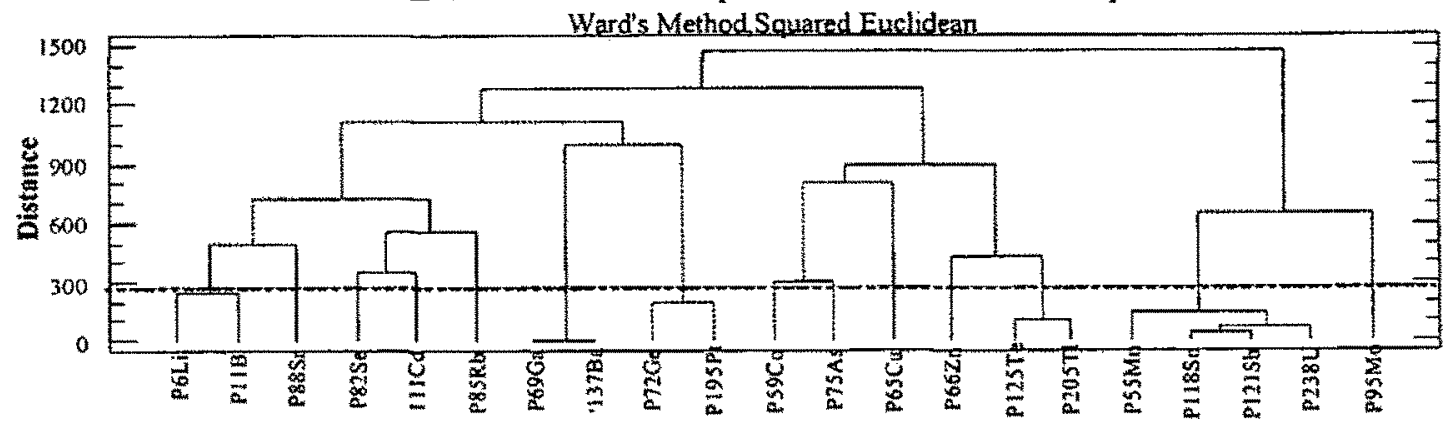

Figure 3 : Analyse en composantes principales et classification hiérarchique ascendante dans les urines.

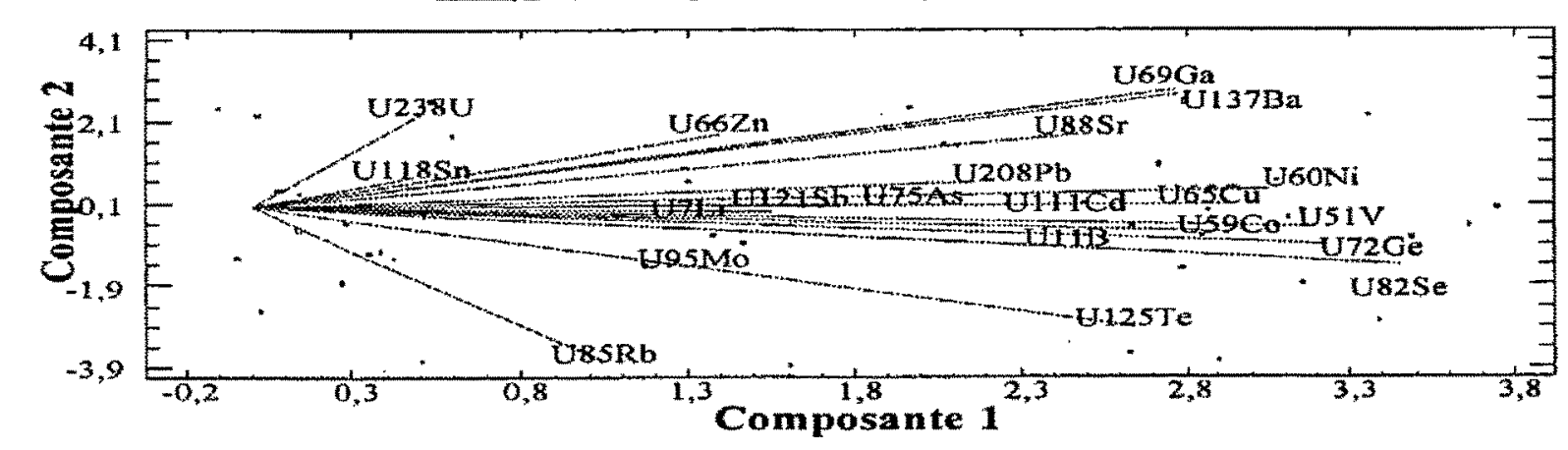

Classification hiérarchique ascendante dans les urines

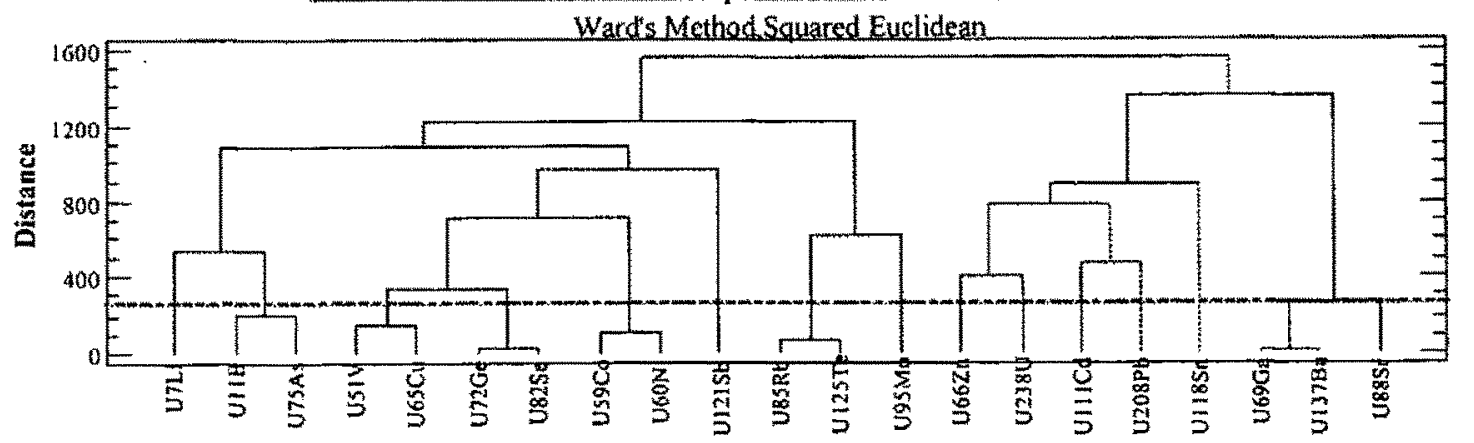


Tableau V

\begin{tabular}{|c|c|c|c|c|c|}
\hline 100 témoins & LD & Moyenne & $\begin{array}{c}\text { Moyenne } \\
+/-2 \text { écart-types }\end{array}$ & Médiane & $\begin{array}{c}\text { Percentile } \\
(\mathbf{5 \%}-\mathbf{9 5} \%)\end{array}$ \\
\hline $\begin{array}{c}\text { Lithium } \\
\text { (urines) }\end{array}$ & 0,01 & 45 & -205 à +295 & 12 & $4,6-219$ \\
\hline $\begin{array}{c}\text { Aluminium } \\
\text { (plasma) }\end{array}$ & 2,3 & 9,0 & $-42 \mathrm{à}+63$ & 3,1 & $1,2-17,3$ \\
\hline
\end{tabular}

$\mathrm{LD}=$ limite de détection - Les résultats plasmatiques et urinaires sont respectivement exprimés en $\mu \mathrm{g} / \mathrm{L}$ et $\mu \mathrm{g} / \mathrm{g}$ de créatinine.

d'un élément s'accompagnant également en règle générale d'une augmentation pour d'autres métaux.

Nous avons également recherché au travers d'interprétations combinées si l'on pouvait trouver des liens à la fois entre les divers éléments et les milieux biologiques. Un trio sanguin a été mis en évidence : l'antimoine, l'étain et l'uranium. Ces trois métaux montrent tous une excellente corrélation entre leurs concentrations plasmatiques et sanguines respectives. Cette constatation est confirmée par l'analyse en composantes principales ainsi que par les dendrogrammes. $\mathrm{Si}$ l'on considère la valeur des risques de première espèce pour ces corrélations, l'ordre de grandeur est proche du dix millième. Il semble donc que cette corrélation ne soit pas le fruit d'un pur hasard mais plutôt d'une similitude dans les processus de régulation. En effet, on constate que pour un élément très lourd - supposé toxique - tel que l'uranium, il n'y a pas de phénomène d'accumulation car les individus âgés n'ont pas les concentrations les plus élevées. De vrais jumeaux ont également été identifiés : le baryum et gallium. Les résultats obtenus par la classification hiérarchique ascendante montrent que ces deux métaux sont, dans les trois milieux biologiques, toujours les premiers à s'apparier. Par ailleurs, dans le plasma, comme dans le sang total, la concentration du baryum est liée à celle du gallium avec un risque de première espèce presque nul. Ceci est d'autant plus inattendu qu'ils ne font pas partie de la même série atomique, n'ont pas la même valence et ont des masses très différentes.

\section{Conclusion}

Outre le gain de temps considérable grâce à une analyse multiélémentaire, le principal avantage de l'utilisation de l'ICP-MS est du point de vue clinique, l'analyse simultanée d'un grand nombre d'éléments avec une grande sensibilité comme le montrent les seuils de détection obtenus. Chez les cent sujets témoins, l'analyse statistique fine des résultats permet d'exploiter au maximum la puissance de l'ICP. L'intérêt de la mesure simultanée des métaux et métalloïdes dans le sang total, le plasma et les urines offre des perspectives nouvelles. Ainsi, il s'avère possible de mettre en évidence non seulement une intoxication aiguë ou chronique par l'un des éléments, mais également de mieux surveiller les expositions professionnelles, domestiques ou environnementales, de prévenir tout risque d'intoxication et de mettre en évidence un empoisonnement. Comparée aux autres techniques de dosage, telles que la spectrophotométrie d'absorption atomique, l'ICP-MS représente une véritable avancée dans le domaine de l'analyse toxicologique y compris dans la recherche des causes de la mort. La cadence analytique élevée, la grande sensibilité et la précision des mesures offrent de nouveaux champs d'application en médecine humaine.

Cet équipement a été acquis grâce à un financement de l'Association pour la Fondation Charles Nicolle Rouen - France (Président Professeur Eric MALLET).

\section{Références}

1. Goullé J.P., Mahieu L., Castermant J., Neveu N., Lainé G., Nouveau M.P., Gehanne R., Bouige D., Lacroix C. Validation d'une technique de dosage multiélémentaire des métaux par ICP-MS dans les milieux biologiques. Ann. Toxicol. Anal. $2003 ; 15: 271-80$. Correctif dans Ann. Toxicol. Anal. $2004 ; 16: 257-60$.

2. Lebart L., Morineau A., Piron M. Statistique exploratoire multidimensionnelle. Paris : Dunod, 1995.

3. Apostoli P., Cortesi I., Mangili A. et al. Assessment of reference values for mercury in urine: the results of an italian polycentric study. Sci. Total Environ. $2002 ; 289$ : 13-24.

4. Chappuis $\mathbf{P}$. Techniques d'analyse des oligo-éléments chez l'homme. Ed. TECH et DOC : Ed. Méd. Int., 1995.

5. Pineau A., Guillard $O$. Techniques d'analyse des oligoéléments chez l'homme «volume 2 ». Ed. TECH et DOC : Ed. Méd. Int., 2001.

6. Lauwerys R.R. Principales substances inorganiques et organo-métalliques. Toxicologie Industrielle et intoxications professionnelles. Ed. Lauwerys, $2000: 131-328$.

7. Garnier R. Produits industriels, éléments in : Bismuth C., ed. Toxicologie clinique. Flammarion, 2000: 555-699. 